\title{
Arthroscopic micro fracture and intra articular whole bone marrow injection for the treatment of articular cartilage lesions in the knee joint.
}

\author{
Firas A. Alobidi*
}

\author{
FICMS(ortho.)
}

Abstract:

J Fac Med Baghdad 2016; Vol.58, No.1 Received Jan. 2016 Accepted Fab. 2016

Background: Articular cartilage lesions in the knee joint are frequently seen,its treatment represents a challenge for orthopedic surgeons especially in young age patients and if left untreated,then they can progress to advanced osteoarthritis that may affect the functional status of the patients.Many treatment modalities had developed for these lesions, in the current study arthroscopic micro fracture plus intra articular whole bone marrow(WBM) injection was used as modality of treating these lesions.

Objectives: to assess the effectiveness of arthroscopic micro fracture plus WBM intra articular injection as a treatment modality for knee articular cartilage lesions.

Patients and Methods : a prospective experimental study was performed on 30 patients (14 females, 16 males ) with a mean age of 38.6 years who had chondral lesions in their knee joint that failed to respond to conservative treatment.Arthroscopic micro fracture plus WBM intra articular injection was done to them. The functional assessment of the patients and their knee pain were evaluated by modified Cincinnati knee rating score (CKRS) and Visual Analogue Scale (VAS) respectively, both preoperatively and six months postoperatively. These results were compared.

Results: there was statistically significant improvement in a modified CKRS and VAS six months after surgery.26 patients out of 30 patients $(86.7 \%)$ were improved.

The improvement was statistically significant for those patients $\leq 40$ years of age and also for those above 40 yeas of age, but it was more pronounced in those patients $\leq 40$ years.

Conclusion : Arthroscopic micro fracture plus intra articular WBM injection is an effective treatment modality for small,well defined,full thickness chondral lesions in the knee joint especially for those $\leq 40$ years old on short term follow up. A long term follow up is needed.

Key words : Arthrospoic + micro fracture + bone marrow injection + chondral knee lesions.

\section{Introduction:}

The knee articular cartilage lesions are frequently seen pathology (1). Aroen et al (2) showed articular cartilage defects in more than $60 \%$ of their arthroscopic knee surgeries. The lesions were also observed in about $50 \%$ of arthroscopic anterior cruciate ligament reconstruction surgeries(3). Because the articular cartilage is avascular with low mitotic activity and has poor spontaneous healing potential, its lesion is difficult to treat and can progress to symptomatic osteoarthritis and affects the patients functional level(4). The management of symptomatic articular cartilage lesions in the knee joint represents a treatment dilemma, especially in young and active people(1).In the past these lesions were treated conservatively e.g. anti inflammatory medications, job modification and intra articular injection of Steroids or viscosupplements (hyaluronic acid), but this type of treatment failed to cause cartilage healing and it only alleviated the symptoms and when the functional level deteriorated, an osteotomy or total knee replacement the major treatment options(5).Recently two strategies of treatment

\footnotetext{
*Dept. of Surgery, Baghdad university/Medical college. E-mail: firas75ortho@yahoo.com
}

have been developed, these are either reparative treatment (e.g. micro fracture ) in which the articular cartilage lesions heal with another type of cartilage called fibrocartilage which does not have the same mechanical characteristics of the original articular cartilage ( hyaline cartilage ), or restorative treatment (e.g. osteochondral autograft or allograft transplantation,and autologous chondrocyte implantation) in which the healing cartilage is much more similar to hyaline cartilage than in reparative treatment, but this strategy of treatment is usually more costly, more surgically demanding,more time consuming and more invasive than reparative type(6). The micro fracture technique is done by creating small holes in the subchondral bone plate of the the lesion which leads to clot formation and then subsequent differentiation to fibrocartilage(1). On the other hand, some authors had reported the benefits of the WBM injection into the knee joints with degenerative lesions(7). In the current study the combination of arthroscopic micro fracture and WBM intra articular injection for the articular cartilage lesions in the knee joint were evaluated from functional improvement point of view and knee pain improvement. 


\section{Patients and Methods:}

A prospective experimental non randomized study was carried out on 30 patients ( 14 females, $46.7 \%$, and 16 males, $53.3 \%$ ) with a mean age of 38.6 years ranging from 23 to 53 years who had evidence of articular cartilage lesions with or without meniscal lesions or other pathologies in their knee joints on clinical and radiological (MRI) examinations that did not respond to conservative treatment and required arthroscopic surgical intervention during the period from April 2012 to May 2015.Patients with fixed flexion deformity of the knee joint or limited range of knee movement, varus or valgus knee deformity of more than 5 degrees, knee instability due to ligament injuries, those who received knee intra articular steroids injection less than 6 weeks before surgery, those with degenerative lesions that are poorly demarcated and had no well defined edges, those with focal cartilage lesions more than 2 Square Centimeter when seen intraoperatively, those with chondral lesions on tibia and femur that face each other ( kissing lesions ), those with knee injury of less than 4 weeks before surgery, those with patellar malalignment or instability, those with avascular necrosis in the knee joint or osteochondritisdissicans, those with chondral lesions that do not reach the underlying bone 1.e. grade zero, one and two according to Outerbridge classification system of chondral lesion(8), table 1, and any patient with postoperative complications (e.g. arthrofibrosis and infection) were excluded from this study.A 30 patients(14 females and 16 males) were met the inclusion criteria and were involved in this study.

Table 1 :Outerbridge grading of the articular cartilage lesions.

\section{$0=$ Articular cartilage is normal}

$1=$ There is softening, swelling, or blistering of the cartilage.

$2=$ Partial thickness fissures that are less than 1 Centimeter in diameter

$3=$ Full thickness fissures that reach to subchondral bone and are larger than 1

Centimeter in diameter

\section{$4=$ The subchondral bone is exposed.}

All the involved patients were optimized to surgery and admitted to hospital 1 day before surgery.Preoperative functional assessment of patients knee joints was performed using the modified CKRS (9), which consists of 8 different sections ( pain intensity, swelling, giving way, overall activity level, walking, stairs, running activity, and jumping or twisting), the maximum score is 100 and the lowest score is 6.It is graded according to the scores (10) as poor ( $<30)$, fair (30-54), good (55-79) and excellent $(\geq 80)$, and the patients knee pain was measured by using VAS (11), figure 1 .

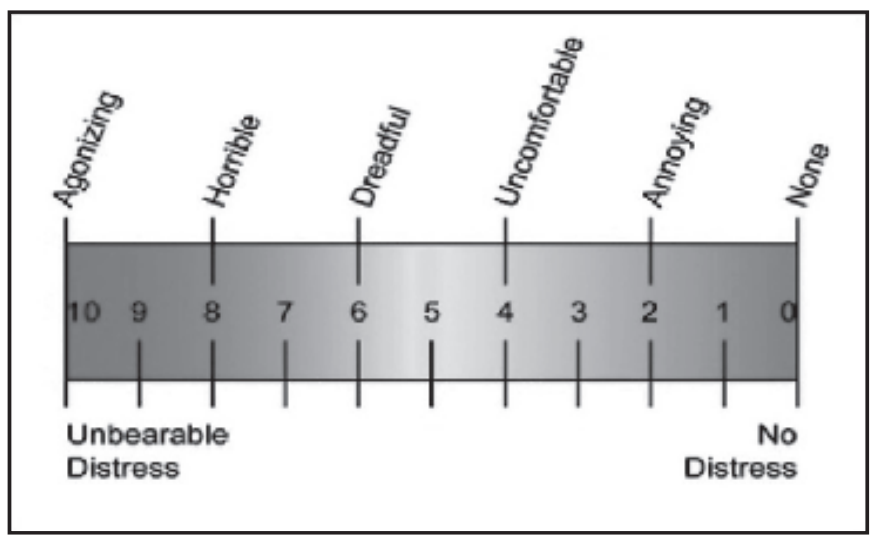

Figure 1: VAS(12).

Prophylacting intravenous antibiotics were given to all patients one hour before skin incision, general or regional anesthesia was used, pneumatic tourniquet was applied and arthroscopic surgery started with diagnostic tour,meniscal lesions were treated by partial meniscectomy and shaving, then micro fracture of the lesions in the articular cartilage was performed according to the procedure of Steadman et al (13) which includes debridement of the lesion by shaver to have stable and well defined margins of normal cartilage in order to prevent any propagation of the injury, then removal of the deep calcified layer of articular cartilage in the lesion by shaver and small curette to expose the underlying bone, then using awl to create a small holes in the subchondral bone of the lesion 3-4 Millimeters apart and down to a depth of about 4 Millimeters, lastly the arthroscopic pump pressure was reduced to confirm bleeding and fat droplets release from the micro holes. Figure 2 shows the well defined edges and exposed bone after debridement and shaving of the articular lesion in the medial femoral condyle of a patient involved in this study.Figure 3 shows the micro holes created in the same lesion in figure 2, figure 4 represents the arthroscopic picture of the same chondral lesion in figure 2 after one year of the micro fracture surgery, the fibrocartilage is seen filling the defect, this patient developed symptomatic meniscal tear after one year of her first surgery and arthroscopic meniscectomy was done to her. 


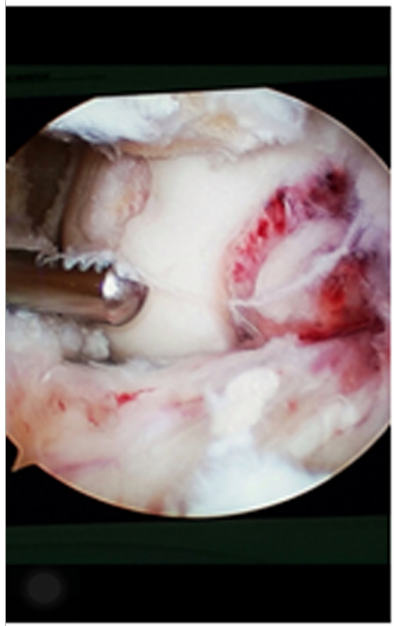

Figure 2 chondral lesion

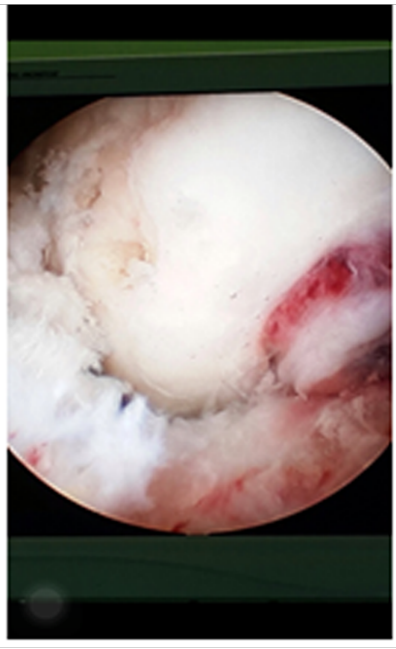

Figure 3 Micro fracture

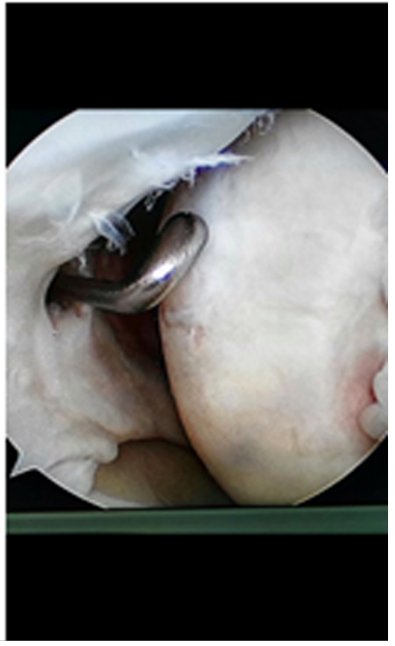

Figure 4 healing by fibrocartilage
The cartilage defect measurement and other measurement were done by the use of arthroscopic probe after calibrating it.After completing micro fracture procedure the irrigation fluid was evacuated from the knee joint and the arthroscopic sleeve was withdrawn,then the whole bone marrow was aspirated from upper anterolateral part of the tibia of the same limb by doing small skin incision (about 2 Centimeters length) and then penetrating the bone cortex by small diameter drill, then the 10 cubic centimeters(CC) syringe that contains heparin( 2000 units in 1CC) was passed through drill hole to aspirate about $10 \mathrm{CC}$ of the WBM and then this was directly injected to the affected knee joint as shown in figure 5 and 6 for one patient in this study, then suturing of skin incisions and dressing were done.

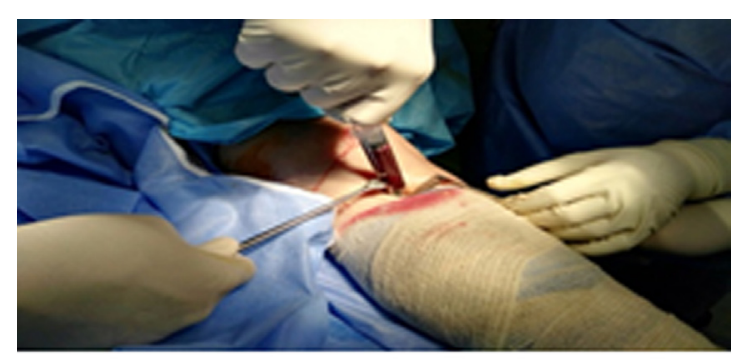

Figure 5 Aspirating WBM

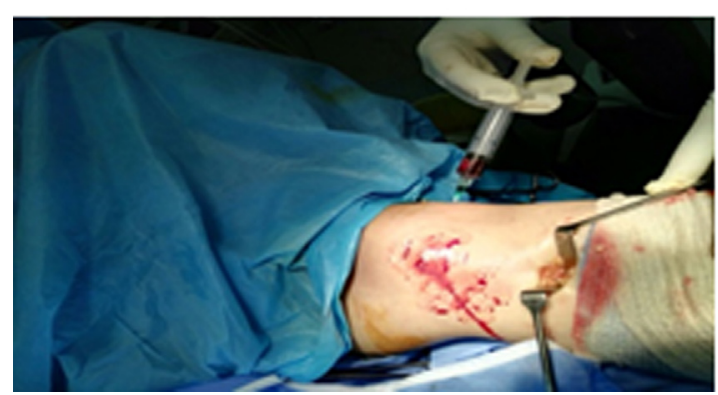

Figure 6 Injecting WBM
The postoperative analgesia and rehabilitation period was the same for all patients, full passive motion of the operated knee joint was gradually practiced about 6 hours per day for the first 6 postoperative weeks and during this period the weight bearing was not allowed, then partial weight bearing was allowed over the next 6 weeks and after that gradual increase until reaching full weight bearing. There was no significant postoperative complications.At 6 months postoperatively the functional assessment of patients and their knee pain were reevaluated by using modified CKRS and VAS respectively. Then the preoperative and postoperative results were compared statistically.

\section{Results:}

30 patients ( 14 females, $46.7 \%$ and 16 males, 53.3\% ) were involved in this study.26 ( $86.7 \%$ ) patients had improvement in their VAS and modified CKRS scores and 4 patients showed no improvement in these scores six months after surgery. student t test was used.

For all patients :

The mean of preoperative and postoperative modified CKRS were 38.9 and 77.3 respectively. $\mathrm{P}$ value is $<0.00001$ (the results is significant at $\mathrm{p} \leq 0.05$ ).

The mean of preoperative and postoperative VAS were 6.9 and 3.06 respectively. $\mathrm{P}$ value is $<0.00001$.

For those patients with $\leq 40$ years of age ( they were 18 patients) :

The mean of modified CKRS (Table 2) was 37.1 preoperatively and 81.8 postoperatively ( $\mathrm{P}$ value is $<0.00001$ ).

The mean of VAS was 7.05 preoperatively and 2.55 ( P value is $<0.00001$ ). Table 4

For those patients with $>40$ years of age ( they are 12 
patients):

The mean of modified CKRS (Table 3 ) was 41.6 preoperatively and 70.4postoperatively ( $\mathrm{P}$ value is $<0.00001$ ).

The mean of VAS was 6.75 preoperatively and 3.83 postoperatively ( $\mathrm{P}$ value is $<0.00001$ ). Table 4

Regarding modified CKRS, the mean difference between preoperative and postoperative results for those $\leq 40$ years old is 44.7 ( 81.8 - 37.1 ), while for those $>40$ years old is 28.8
(70.4 - 41.6 ). so the improvement in those $\leq 40$ years old is better.

Regarding VAS, the mean difference between preoperative and postoperative results for those $\leq 40$ yeas old is 4.5 ( 7.05 -2.55 ), while for those $>40$ years old is 2.9 ( $6.75-3.83)$.so the improvement in those

$\leq 40$ years old is better.

Table 2 : Modified CKRS before and 6 months after surgery for those $\leq 40$ years old.

\begin{tabular}{cccccccccccccccccccc}
\hline Patient number & 1 & 2 & 3 & 4 & 5 & 6 & 7 & 8 & 9 & 10 & 11 & 12 & 13 & 14 & 15 & 16 & 17 & 18 \\
\hline Before surgery & 47 & 43 & 40 & 35 & 30 & 31 & 28 & 30 & 49 & 55 & 33 & 39 & 30 & 32 & 38 & 41 & 38 & 29 \\
\hline After surgery & 45 & 87 & 85 & 82 & 79 & 85 & 86 & 86 & 88 & 79 & 85 & 85 & 87 & 85 & 82 & 78 & 85 & 85 \\
\hline
\end{tabular}

Table 3 : Modified CKRS before and 6 months after surgery for those $>40$ years old.

\begin{tabular}{ccccccccccccc}
\hline Patient number & 1 & 2 & 3 & 4 & 5 & 6 & 7 & 8 & 9 & 10 & 11 & 12 \\
\hline Before surgery & 34 & 57 & 38 & 44 & 45 & 44 & 34 & 37 & 56 & 30 & 50 & 30 \\
\hline After surgery & 75 & 58 & 76 & 72 & 73 & 75 & 80 & 69 & 55 & 76 & 50 & 86 \\
\hline
\end{tabular}

Table 4 : Histogram showing the results for those patients $\leq 40$ years and $>40$ years old.

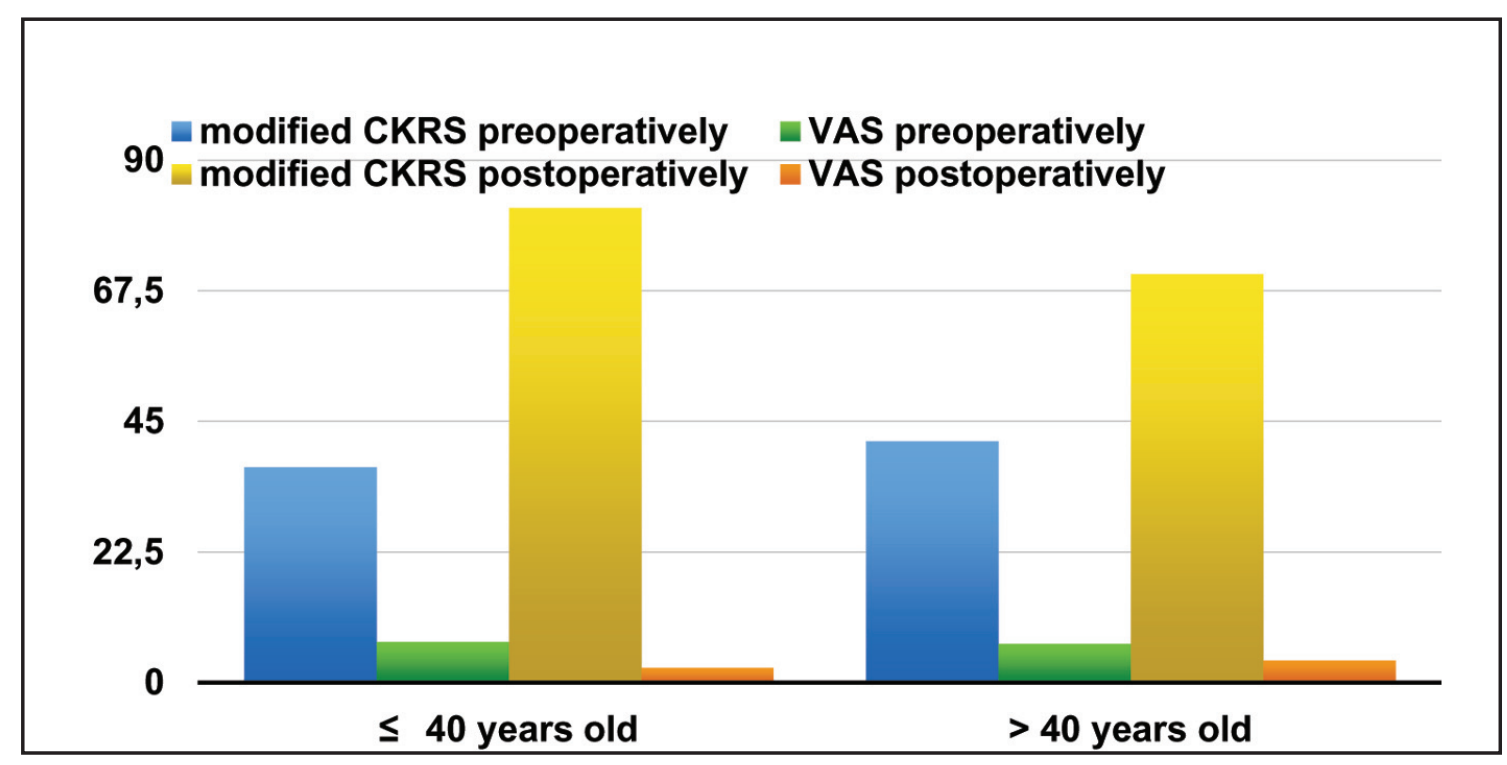

\section{Discussion:}

The micro fracture technique of Steadman et al(13) is a modification of the micro drilling technique that was advocated by Pridie(14) at 1959 , both of them are marrow stimulating techniques that involve subchondral bone plate perforations in the cartilage defects that leads to clot formation in these defects, and subsequent proliferation of pluripotent marrow- derived mesenchymal cells which can produce fibrocartilage repair(15).The advantages of micro fracture over micro drilling includes less thermal necrosis and damage to osteocyte around the holes, more rough surface for the healing tissue to be adhered, and more easily to perforate certain lesions perpendicularly with a curved awl than a drill in arthroscopic surgery(16). The 3-4 Millimeters distance between the holes of 
micro fracture was maintained to preserve subchondral bone plate and its function(1),while the 4 Millimeters perforation depth was performed to ensure more access to bone marrow and so more repair(4). The short term clinical results of micro fracture in current study showed improvement of knee function and decrease in knee pain in $86.7 \%$ of patients( 26 patients out of 30 patients) which is comparable with several other studies that had shown improvement in the knee function in 70 to $95 \%$ of patients after micro fracture $(17,18,19)$. Kreuz et al(20) showed that the outcome of micro fracture is age dependent and that the age of 40 years and below represents the best prognostic factor for repair and healing.In the current study the improvement in those patients with 40 years of age or less was more than those with more than 40 years old, this was statistically significant depending on the mean difference, this may be due to differences in quality and quantity of metabolic activity in the healing cartilage between different age groups(21).In the current study in spite of that the improvement was more in those patients below 40 years of age than those with 40 years old or less, both age groups showed statistically significant improvement l.e. even those with more than 40 years old had also statistically significant improvement and this might be explained by adding the whole bone marrow injection to micro fracture technique, however this improvement was on short term follow up(six months).Kreuz et al (20) found that deterioration started 18 months after surgery and was significantly shown in those patients with more than 40 years old, and Steadman et al(13) had improvement in $75 \%$ of patients with 3 to 5 years of follow up.so long term follow up is needed for patients in the current study.Arthroscopic micro fracture plus whole bone marrow injection technique is minimally invasive, cheap, and less surgically demanding than some other surgical modalities( e.g. autologous chondrocyte implantation and osteochondral allograft transplantation) for the treatment of chondral lesion in the knee joint, but it mainly forms fibrocartilage that is less durable than hyaline cartilage. However,certain studies(22) that compared the autologous chondrocyte implantation to micro fracture for the treatment of small chondral lesions ( less than 3 square Centimeters) had found similar radiographic and clinical outcomes. The short term follow up and relatively small sample size represent the main limitations in the current study.

\section{Conclusions :}

This study showed statistically significant improvement of small size ( less than 2 secure centimeters), well defined, full thickness knee articular cartilage lesions after arthroscopic micro fracture plus whole bone marrow intra articular injection on short term follow up, and this improvement was statistically significant in both patients aged more than 40 years and patients aged less than 40 years with more pronounced improvement in those aged less than 40 yeas.

\section{References}

1-Mithoefer B, Williams R, Warren R, Potter H, Spock C, Jones $E$ et al.The micro fracture technique for the treatment of articular cartilage lesions in the knee. J Bone Joint Surg 2005; 87-A(9) : 1911-1920.

2-Areon A, Loken S, Heir S, Alvik E, Ekeland A, Granlund OG et al. Articular cartilage lesions in 993 consecutive knee arthroscopies.Am J Sports Med. 2004;32 : 211-215.

3-Piasecki DP, Spindler KR, Warren TA, Andrish JT, Parker $R D$. Intra articular injuries associated with anterior cruciate ligament tear : findings at ligament reconstruction in high school and recreational athletes. An analysis of sex-based differences. Am J Sports Med.2003; 31 : 601-605.

4-Chen H, Hoemann C, Sun J, Chevrier A, Mckee M, Shive $M$ et al. Depth of subchondral perforation influences the outcome of bone marrow stimulation cartilage repair. Journal of Orthopedic Research 2011;29:1178-1184.

5-Detterline A, Goldberg S, Bach B, Cole B. Treatment options for articular cartilage defects of the knee. Orthopedic Nursing 2005;24(5) : 1-6.

6-Falah M, Nierenberg G, Soudry M, Hayden M, Volpin G. Treatment of articular cartilage lesions of the knee. Internationalorthopaedics( SICOT) 2010;34:621-630.

7-Orlofsky A, Hauser R. Regenerative injection therapy with whole bone marrow aspirate for degenerative joint disease : A case series. Clinical Medicine Insights : Arthritis and Musculoskeletal Disorder 2013;6 : 65-72.

8-Browne JE, Branch TP. Surgical alternative for treatment of articular cartilage lesions. J Am AcadOrthopSurg 2000;8(3) : 180-189.

9-Noyes FR, Barber SD, Mooar LA. A rationale for assessing sports activity levels and limitations in knee disorders. ClinOrthopRelat Res. 1989; Sep; 246 : 238-249.

10-Bentley G, Biant LC, Carrington RW, Akmal M, Goldberg A, Williams AM et al. A prospective randomized comparison of autologous chondrocyte implantation versus mosaicplasty for osteochondral defects in the knee. J Bone Joint Surg Br. 2003 Mar; 85(2) : 223-230.

11-Huskisson EC.Management of pain. J. Rheumatol 1982; 9(5) : 768-769.

12-Shah M, ShivaswamyS, Jain Sanjay, Tambwekar S. A clinical comparison of pain perception and extent of area anesthetized by wand and a traditional syrinage. Journal of Indian Society of Periodontology 2012; 16(2) : 207- 212.

13-Steadman JR, Rodkey WG, Rodrigo JJ. Micro fracture : 
surgical technique and rehabilitation to treat chondral defects.

ClinOrthopRelat Res. 2001;391: s 362-369.

14-Pridie KH.A method of resurfacing osteoarthritic knee joints. J Bone Joint Surg \{Br\} 1959; 41-B : 618-619.

15-Chen H, Sun J, Hoemann C, Lascau-Coman V, Ouyang W, Mckee M. Drilling and microfracture lead to different bone structure and necrosis during bone-marrow stimulation for cartilage repair. J Orthop Res 2009;27:1432-1438.

16-Smith G, Knutsen G, Richardson J. A clinical review of cartilage repair techniques. J Bone Joint Surg $\{B r\} 2005$; 87$B(4): 445-449$.

17-Knutsen G, Engebretsen L, Ludvigsen TC, Drogset JO, Grontvedt T, Solheim E et al. Autologous chondrocyte implantation compared with micro fracture in the knee. A randomized trial. J Bone Joint Surg Am. 2004; 85 : 455-464.

18-Steadman JR, Briggs KK, Rodrigo JJ, Kocher MS, Gill TJ, Rodkey WG. Outcomes of micro fracture for traumatic chondral defects of the knee : average 11 year follow up. Arthroscopy. 2003;19:477-484.

19-Gobbi A, Nunag P, Malinowski K. Treatment of full thickness chondral lesions of the knee with micro fracture in a group of athletes. Knee Surg Sports TraumatolArthrosc. 2005; $13: 213-221$.

20-Kreuz PC, Erggelet C, Steinwachs MR, Krause SJ, Lahm A, Niemeyer $P$ et al. Is micro fracture of chondral defects in the knee associated with different results in patients aged 40 years or younger? Arthroscopy 2006; 22(11): 1180-1186.

21- Martin JA, Buckwalter JA. The role of chondrocyte senescence in the pathogenesis of osteoarthritis and in limiting cartilage repair. J Bone Joint Surg Am. 2003; 85(2) :106-110. 22-Van Assche D, Staes F, Van caspelD, Vanlauwe J, BellemansJ , Saris D et al. Autologous chondrocyte implantation versus microfracture for the knee cartilage injury : a prospective randomized trial, with 2 year follow up. Knee Surg Sports TraumatolArthrosc. 2009; 18 (4) : 486-495. 